\title{
Using XML Files to Document the User Interfaces of Applications
}

\author{
Mohammad Tubishat, Izzat Alsmadi, and Mohammed Al-Kabi \\ Yarmouk University, Jordan \\ mtubishat@yu.edu.jo,ialsmadi@yu.edu.jo,mohammedk@yu.edu.jo
}

\begin{abstract}
User interfaces represent a major part in software products. Generally, it is difficult to document the content and the structure of the user interface. However, there is a need to save and preserve the overall state and structure of the user interface for later communication and modifications in requirements, design and implementation. The Graphical User Interface (GUI) storage format should be universal and can be transferred from one application to another (such as XML). GUI states are usually defined as the overall combination of properties of all the components or widgets of the GUI.
\end{abstract}

This paper suggests an alternative back end representation of user interfaces and their state from their original format within the applications. The user interface model is transformed to a schema saved in an XML file. The schema structure represents the user interface state. This state considers only the structure of the user interface and ignores controls' specific properties. XML format is widely used and accepted by many applications. It is the infrastructure language for web pages and database management systems. User interface documentation is useful for future evaluation and comparison, useful for stakeholders' communication. It is also for some GUI testing activities such as regression testing where we trigger testing if there is a change in the user interface.

Index Terms - GUI states, user interface testing, modeling, XML, and state charts.

\section{INTRODUCTION}

In new software applications, the user interface takes a large portion of the overall software. User interfaces are also always changing and evolving. User interfaces can be documented in terms of prototypes, screenshots, descriptions, etc. Several applications use the user interface realization for services such as undo, redo, restore, etc.

State charts are used to generate tests in modelbased architectures. They describe state transitions of objects with states. Model checking is a technique used for verifying a system composed of concurrent finitestate machines. State machines should be finite as model checkers need to exhaustively explore the entire state space of the state machine.

In user interface testing, the general accepted approach defines a GUI state through all GUI components and their properties. This means that a change in a single property of a single GUI control or widget causes the GUI state to be changed [1]-[2][3]-[4]-[5]-[6]-[7]. Defining the GUI states by the combination of all its widgets and properties produces a very large number of possible GUI states. Changing any single property in any widget causes the whole GUI state to be different.

In modern applications, the ability to save and control the GUI state is very useful in several features such as: Undo, redo, animation, hide, show, enable, disable, etc. For example, a user wants to be able to undo an action or actions and remove their effect all over the application. If a user is using an application and power failure is suddenly occurred, the application should have the ability to save the last GUI state to allow it to be recovered.

In this project, we propose a different way to define the GUI state. We suggest defining the GUI state through the XML file that represents the GUI. The reason for doing this is to automate the way we compare two different GUI states (through XML files' comparison) and to save the GUI state in a universal format that can be stored for later stages in the projects or transferred to another application.

When using XML to store the GUI tree, a new definition is introduced for a GUI state. Rather than assuming that the GUI state depends on each property for each control of the whole application, we define the GUI state as the hierarchy that is embedded in the XML tree. A GUI state change here means a change in any parent-child relation, or any change in the specific properties that are parsed.

This new definition produces an effective reduction in GUI states. For example a small application such as Notepad, can have more than 200 controls, each of which may have more than 40 properties, this produces $200 * 40=8000$ states. Any property or control from the 8000 controls and properties triggers the state change. In our assumption, 200 controls will have 200 parent-child relations (or less). Each control has less than 10 relevant parsed properties. The total possible GUI states are reduced to 2000 or a $75 \%$ total reduction.

In testing, we usually use the evolution or modification of the user interface state as a reason to trigger or reapply several testing processes. For 
example, In GUI test automation, GUI scripts should be regenerated or revisited upon GUI state changes (regression testing). As such activities can be expensive; we need to trigger them only when there is a good reason to do that.

Each GUI has many forms, each form has many controls, and each control has many properties. The general definition used in many literatures [1]-[2]$[3]-[4]-[5]-[6]-[7]$ that a change in any control property causes a GUI state change means that the number of states in any GUI, is very large even for a small application.

The GUI state change is used in testing to trigger some testing activities such as integration or regression testing. The number of possible GUI states affects the space of creating test cases which means that the more possible states we have, the more test cases we need to generate for test adequacy.

\section{RELATED WORK}

An object state is the condition(s) of that object in a given time. A given state for an object defines the events that may affect it at this time, and the next possible states. A GUI state is described in terms of the specific objects or controls it currently contains, and the values of their properties $[8,9]$. The information of a GUI state at any particular time is important for testing.

Saving the GUI to a GUI state file is investigated by several papers [10]-[11]. The GUI state can be saved and retrieved from such files. This facilitates the usage of some services such as: undo, simulation, and testing, and the storage of the current state.

In literature, usually, there is an ambiguity between the application and the user interface states. Despite the fact that those two states are related, yet they are not identical. The application state is the state of all the application resources, including the user interface, at any given time.

Currently, there are many user interface description languages used to facilitate communicating with the interface, and the code that sets behind it. This is occurred in different software engineering tasks such as: requirement, design and testing. We will use XAML as an example.

XAML (eXtensible Application Markup Language; pronounced "Zammel") is a new Microsoft Longhorn declarative markup programming language for building applications' user interfaces. Elements in the XAML file are correlated to the GUI objects at run time. XAML utilizes XML hierarchical logic to present the hierarchy of GUI objects. This makes our testing framework matches in principles the approach XAML is taking. It is possible to develop a solution with XAML without developing any code or develop partial XAML/code applications.

The ultimate goal of XAML is to have a standard syntax for describing user interface controls and eventually serializing all GUI components in XAML files. This will be very useful in several ways. In one particular advantage, the UI implementation will be documented in a way that can be easily accessed, edited, and reconstructed. Some other advantages expected from having a standard syntax for UI design and implementation is; the ease of modifying the UI even at run time, the ability to separate UI components from the other layers of the application or separate the UI model from its view, and the ability to reuse the UI or some of its components.

XAML can be used as a UI modeling language to create UI elements that can be implemented in any platform and with any programming language (theoretically). XAML simplified control properties into its type and text only. The created button has default visual presentation through theme styles, and default behaviors through its class design. For testing, this reduces the large amount of possible UI states relative to alternatives.

A control can be presented in XAML using the line;

$<$ Panel1 $>$ <Button Content="OK"/> </Panel1 $>$, where the panel, panell and the button $\mathrm{OK}$ are two XAML elements.

XAML elements (i.e. UI controls) are mapped to .NET types that can be extracted from their assembly. Abstract classes are not mapped to XAML tags.

The second related subject we will discuss is Avalon and Windows Presentation Foundation layer (WPF). WPF is WinFX (i.e. .NET framework 3.0) user interface framework or graphics subsystem platform for Windows client's applications. It is preinstalled in MS Windows Vista operating system. In WPF, control's logic is separated from its appearance that adds flexibility to the way controls can be displayed. WPF content can be hosted in a Win32 window and visa-versa.

\section{GOALS AND APPROACHES}

For software testing, the reason or goal for studying the state of an object at any given time is to know the "scope" of that object, i.e., to know the current actions that may affect the object and the results of those actions. This is important in particular, for transactional processing applications. For example, if a car is in an initial complete "off" state, some actions such as "switch on" are available, while others, such as "accelerate" are not.

Should changing the color of one control in the whole GUI causes a change to the whole state of the GUI?! In other words, will such action disable some events and enable some others?! Maybe it is inaccurate 
to say that in all cases, such change will not have any impact on the overall GUI state, but for the most cases, it will not. To deal with the problem of having large number of GUI states, we have to consider the major ones only.

This customized definition of GUI states is suggested in a GUI test automation framework [12, 13]. The application displays the GUI hierarchy, its controls, and properties.

The tool checks for GUI state changes through comparing the current XML tree file that represents the GUI of the application with the previous one (or any other selected one). The tool can display the GUI hierarchy, its controls, and properties. Testers can then specify the properties that they want them to trigger a GUI state change as this can be different with the different scenarios.

The GUI states comparison is done automatically through the tool. This comparison and checking of the overall GUI state (i.e. GUI structure) is not intended to be used for test case generation. It can be used to trigger the execution of regression testing (In the same way a sensor triggers switching on, or off, an air condition system once it reaches a cut off ,high or low, degrees).

Implementing some actions such as undo, redo, restore, etc using XML files comparison can be straightforward. We can utilize several other advantages of using XML files to represent the GUI structure. One of the challenges in software design and coding is that, especially later in development, developers and other project team members need to have a common ground or form of the application user interface to use for discussion and feedback.

The application user interface can be saved in those files for re-usage and testing. GUI re-use is usually out of context for software developers. However, XML files abstract the GUI structure and save it in a format that can be reused.

Comparing the GUI design and implementation can be achieved automatically if we have the user interface represented through some XML files. Those are some of the advantages sought in using GUI description languages such as XAML and XUL.

\section{CONCLUSION AND FUTURE WORK}

The idea of defining the GUI state as the collection state for all its controls, such that a change of a single property in one control leads to a new state is valid, but is the reason for producing the huge number of possible GUI states. In software testing, we need to prioritize testing and retest the states or conditions that are critical over trying to exhaustingly test all possible GUI states. In GUI testing, we usually use a combination of users and test automation to provide the best testing adequacy or coverage. For GUI test automation in general and regression testing in particular, we are interested to reevaluate and reexecute the test suite in some particular cases and not in every GUI state change.

The automatic comparison and verification of the overall GUI state (i.e. GUI structure) is not intended to be used for test case generation. It can be used to trigger the execution of regression testing in the same way a sensor triggers switching on, or off, an air condition system.

We considered only the standard XML format. In future, we will develop an adaptor to deal with the different XML formats.

\section{REFERENCES}

[1] Memon, Atef. "A comprehensive framework for testing graphical user interfaces". Ph.D. thesis. Department of computer science, university of Pittsburgh. 2001.

[2] Q. Xie. "Developing cost-effective model-based techniques for GUI testing". In proceedings of the international conference of software engineering (ICSE'06). Shanghai, China. Pages: 997 - 1000. 2006.

[3] Memon, Atef, and Q. Xie. "Studying the fault detection effectiveness of GUI test cases for rapidly evolving software". IEEE transactions on software engineering. Volume 31, Issue 10. Pages: 884 - 896. 2005.

[4] Memom, Atef, I Banerejee, and A. Nagarajan. "GUI ripping: Reverse engineering of graphical user interfaces for testing". In proceedings of the 10th working conference on reverse engineering (WCRE'03). Page 260. 2003.

[5] Memon, Atef. "Developing testing techniques for event-driven pervasive computing applications". OOPSLA'04 Workshop on building software for pervasive computing (BSPC'04). Vancouver, Canada. 2004.

[6] Memon, Atef. "GUI testing: pitfall and process. Software technologies". August 2002. Pages 87-88. < http://doi.ieeecomputersociety.org/10.1109/MC.2002.102379 $5>$.

[7] Memon, Atef. "Hierarchical GUI test case generation using automated planning". IEEE transactions on software engineering. Vol 27, number 2. Pages: 144-155. 2001.

[8] Karam, Karam, Sergiu M. Dascalu, and Rami H. Hazimé. "Challenges and opportunities for improving codebased testing of graphical user interfaces". Journal of Computational Methods in Science and Engineering. 2006. Pages: $379-388$.

[9] A. M. Memon, I. Banerjee, and A. Nagarajan. "What test oracle should I use for effective GUI testing?" In Proceedings of 18th IEEE International Conference on Automated Software Engineering, pages 164-173, 2003.

[10] Tung, Ramona, and Kevin Tong. "A multi-mission deep space telecommunications analysis tool: the Telecom Forecaster Predictor". Aerospace Conference Proceedings, 2000 IEEE. Pages: 397-402.

[11] Cheung , Kar-Ming, Ramona Tung, and Charles H. "Development Roadmap of an Evolvable and Extensible 
Multi-Mission Telecom Planning and Analysis Framework". California Institute of Technology

$<$ http://trsnew.jpl.nasa.gov/dspace/bitstream/2014/40407/1/0 3-1405.pdf> 2008.

[12] Alsmadi, I, and Kenneth Magel. "GUI Path Oriented Test Generation Algorithms". In Proceeding of IASTED (569) Human-Computer Interaction. 2007.

[13] Alsmadi, I, and Kenneth Magel. "An Object Oriented Framework for User Interface Test Automation". MICS07. 2007. 